ARTICLE

Received 1 Apr 2015

| Accepted 20 Jul 2015

Published 24 Aug 2015

DOl: $10.1038 /$ ncomms 9112

OPEN

\title{
Electrical detection of ortho-para conversion in fullerene-encapsulated water
}

\author{
Benno Meier ${ }^{1}$, Salvatore Mamone ${ }^{1}$, Maria Concistrè', Javier Alonso-Valdesueiro ${ }^{1}$, Andrea Krachmalnicoff ${ }^{1}$,
} Richard J. Whitby ${ }^{1} \&$ Malcolm H. Levitt ${ }^{1}$

Water exists in two spin isomers, ortho and para, that have different nuclear spin states. In bulk water, rapid proton exchange and hindered molecular rotation obscure the direct observation of two spin isomers. The supramolecular endofullerene $\mathrm{H}_{2} \mathrm{O} @ \mathrm{C}_{60}$ provides freely rotating, isolated water molecules even at cryogenic temperatures. Here we show that the bulk dielectric constant of this substance depends on the ortho/para ratio, and changes slowly in time after a sudden temperature jump, due to nuclear spin conversion. The attribution of the effect to ortho-para conversion is validated by comparison with nuclear magnetic resonance and quantum theory. The change in dielectric constant is consistent with an electric dipole moment of $0.51 \pm 0.05$ Debye for an encapsulated water molecule, indicating the partial shielding of the water dipole by the encapsulating cage. The dependence of bulk dielectric constant on nuclear spin isomer composition appears to be a previously unreported physical phenomenon.

\footnotetext{
${ }^{1}$ School of Chemistry, University of Southampton, Southampton SO17 1BJ, UK. Correspondence and requests for materials should be addressed to B.M. (email: b.meier@soton.ac.uk) or to M.H.L. (email: mhl@soton.ac.uk).
} 
W ater, like dihydrogen, has two different spin isomers, called ortho and para, which have different spin state symmetries. In ortho-water, the spin state of the two proton nuclei is symmetric under particle exchange and the total nuclear spin has quantum number $I=1$. In para-water, the spin state is antisymmetric and has nuclear spin quantum number $I=0$. Water spin isomerism is of relevance to a broad range of scientific fields from nuclear magnetic resonance (NMR) to astrophysics ${ }^{1-5}$, and closely related to long-lived nuclear spin states, which also involve the slow interconversion of nuclear singlet and triplet states 6,7 .

Physical properties of dihydrogen $\mathrm{H}_{2}$, such as heat capacity or thermal conductivity, depend on the concentration of ortho and para spin isomers 8 . Do the spin isomers of water also have different bulk properties? Since water, unlike dihydrogen, possesses an electric dipole moment, the spin isomers of ortho and para water are expected to display a distinct response to electric fields. This effect was predicted theoretically'; and observed in beam experiments ${ }^{10}$, but no bulk properties have been reported.

Although it is feasible to separate ortho- and para-water molecules in rarified molecular beams $\mathrm{s}^{5,10}$, it remains challenging to study the separated isomers in the condensed phase, since rapid proton exchange obscures the spin isomerism in bulk water, and strong intermolecular interactions usually quench the molecular rotation at low temperatures. Spin isomer-enriched water may be captured in an inert gas matrix and studied using infrared spectroscopy 11,12 , but this approach provides little control over the molecular environment.

In contrast, the supramolecular endofullerene $\mathrm{H}_{2} \mathrm{O} @ \mathrm{C}_{60}$, composed of $\mathrm{C}_{60}$ carbon cages that each encloses a single water molecule, forms a well-defined lattice. The synthesis of this material provides macroscopic quantities of a stable substance that contains isolated and freely rotating water molecules ${ }^{13,14}$. It has been studied under a very wide range of physical conditions using various spectroscopic techniques ${ }^{1,2,5}$. Dielectric measurements were made on a single crystal of $\mathrm{H}_{2} \mathrm{O} @ \mathrm{C}_{60}$, but without anticipating, or observing, a dependence on spin isomer composition ${ }^{15}$.

Figure 1a,b shows the molecular structure of $\mathrm{H}_{2} \mathrm{O} @ \mathrm{C}_{60}$ and the four lowest rotational energy levels as determined by neutron scattering, neglecting the observed splitting of the ortho-water ground state ${ }^{1,3}$. The energy levels are similar to those of water in
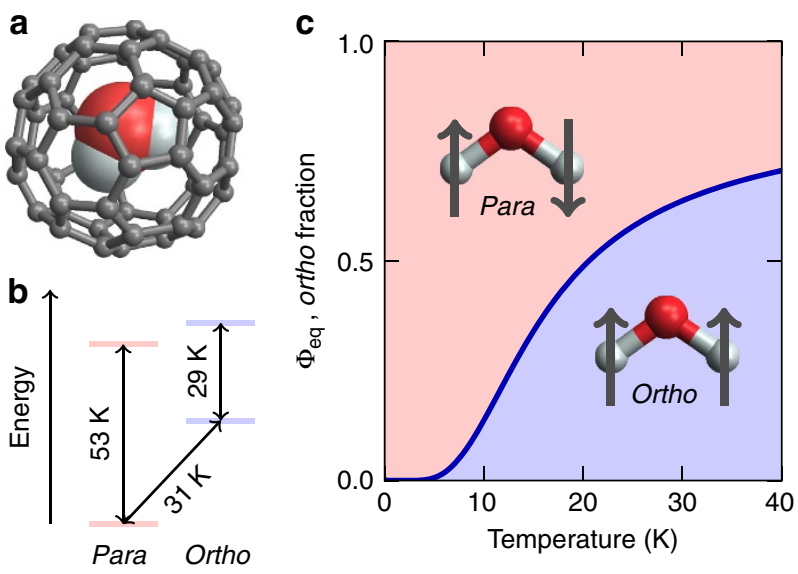

Figure 1 | Ortho and para water inside $\mathbf{C}_{60}$. (a) Molecular structure of the endofullerene $\mathrm{H}_{2} \mathrm{O} @ \mathrm{C}_{60}$ and (b) the lowest rotational energy levels, in temperature units ${ }^{1,3}$. The small splitting of the ortho- $\mathrm{H}_{2} \mathrm{O}$ ground state is ignored for simplicity. (c) Blue line: fraction of ortho-water $\Phi_{\text {eq }}$ as a function of temperature in thermal equilibrium. the gas phase indicating that the water rotation is unhindered even at cryogenic temperatures. The thermal equilibrium fraction of ortho-water molecules as a function of temperature is shown in Fig. 1c, using the energy levels of Fig. 1b and taking into account the degeneracies of the rotational levels ${ }^{2,16}$. The equilibrium fraction changes rapidly in the vicinity of $15 \mathrm{~K}$. Ortho-para conversion may therefore be induced by (i) allowing the sample to reach complete equilibrium at a temperature $>15 \mathrm{~K}$, (ii) rapidly cooling to $<15 \mathrm{~K}$ and (iii) studying the behaviour of the sample as a function of time at the constant low temperature.

Here we demonstrate that the bulk dielectric constant of $\mathrm{H}_{2} \mathrm{O} @ \mathrm{C}_{60}$ depends on the spin isomer composition of the encapsulated water molecules. We find a time-dependent change in dielectric constant at $5 \mathrm{~K}$ that is due to different molecular polarizabilities of the ortho and para ground states. The polarizabilities are extracted from the capacitance data and compared with a theoretical prediction that only requires knowledge of the dipole moment of $\mathrm{H}_{2} \mathrm{O} @ \mathrm{C}_{60}$ and the rotational constants of water. The dipole moment is estimated from a high-temperature measurement of the molecular polarizability and found to be in very good agreement with recent predictions of $0.5 \pm 0.1$ Debye $^{17-19}$.

\section{Results}

Dielectric constant. The dielectric response of water on orthopara conversion is demonstrated with the apparatus shown in Fig. 2 (see Supplementary Methods for details). Three capacitors are measured simultaneously: one is filled with a 4:1 homogeneous mixture of $\mathrm{H}_{2} \mathrm{O} @ \mathrm{C}_{60}$ and $\mathrm{C}_{60}$, one is filled with pure $\mathrm{C}_{60}$, and one is left empty. The apparatus was used to measure (1) the variation of dielectric constant with time at low temperature, which is related to ortho-para conversion and (2) the variation of dielectric constant with temperature above $50 \mathrm{~K}$. The low-temperature experimental data are shown in Fig. 2c-f. Following equilibration at $25 \mathrm{~K}$, the temperature is rapidly decreased to $5 \mathrm{~K}$ and stabilized within $\pm 30 \mathrm{mK}$ for the remaining time of the experiment. The capacitance of the $\mathrm{H}_{2} \mathrm{O} @ \mathrm{C}_{60}$ cell decreases over approximately $50 \mathrm{~h}$. No significant variation is observed for the capacitor filled with pure $\mathrm{C}_{60}$, or the empty capacitor. All measurements are performed at constant frequency and temperature, so that ortho-para conversion is the only process that can account for the decay shown in Fig. 2c. Other possible influences such as geometric drift or gradual penetration of liquid helium into the cell may be discounted since the pure $\mathrm{C}_{60}$ and empty capacitors do not show similar behaviour.

Nuclear magnetic resonance. Proton NMR measurements provide an independent measure of nuclear spin conversion in $\mathrm{H}_{2} \mathrm{O} @ \mathrm{C}_{60}$ since the ${ }^{1} \mathrm{H}$ NMR signal is proportional to the ortho spin isomer fraction $\Phi$. At a temperature of $5 \mathrm{~K}$, the spin isomer conversion takes many hours and follows second-order kinetics ${ }^{2}$. The $600 \mathrm{MHz}$ proton NMR signal amplitude after rapid sample cooling from 25 to $5 \mathrm{~K}$ was measured in a magnetic field of $14.1 \mathrm{~T}$ using a separate apparatus but the same sample batch as the one used for the capacitance measurements. The NMR signal is shown in Fig. 3 (black points). The NMR results show a small but significant ortho fraction persisting even after $40 \mathrm{~h}$ at $5 \mathrm{~K}$. The reasons for the metastable ortho fraction in this sample batch are currently unknown.

The results of NMR and capacitance measurements are compared in Fig. 3. As described in the Supplementary Discussion, the NMR data were shifted in time by $2.2 \mathrm{~h}$ with respect to the capacitance data to take into account the smaller ortho fraction at the time the final temperature is reached in the NMR experiment, compared to the capacitance measurement. 
a

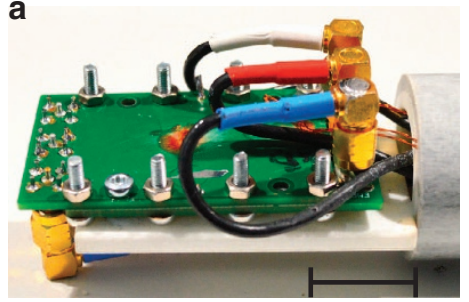

b

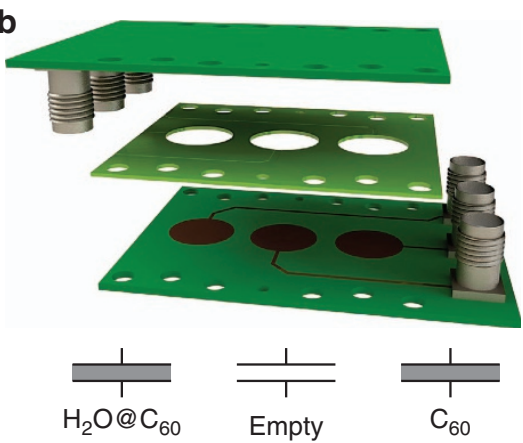

C

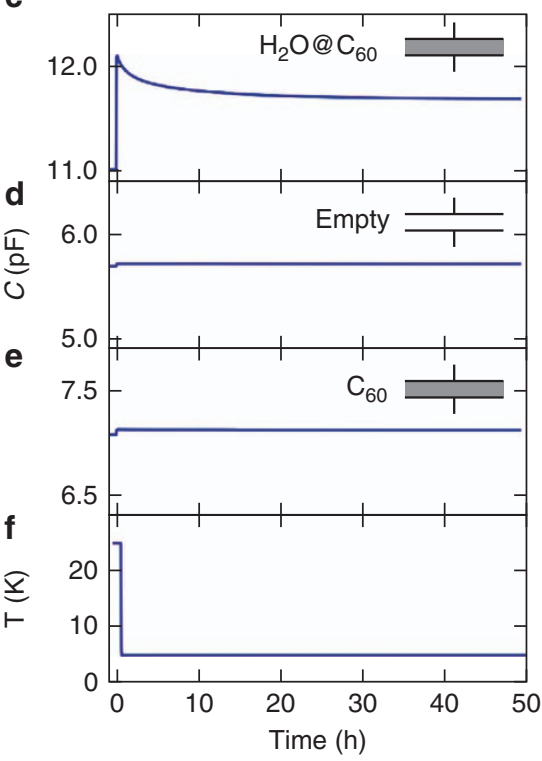

Figure 2 | Detection of ortho-para conversion in $\mathbf{H}_{\mathbf{2}} \mathbf{O} @ \mathbf{C}_{\mathbf{6 0}}$. (a) Photograph and (b) exploded view of the sample cell consisting of two printed circuit boards (PCBs, green) and a $210 \mu \mathrm{m}$ thick G10 spacer used to centre the sample pellets between the electrodes of the two PCBs, forming three independent capacitor cells. Scale bar length in $\mathbf{a}$ is $2 \mathrm{~cm}$. Following a temperature jump from 25 to $5 \mathrm{~K}$ at the time origin, the capacitance of the three cells is measured as a function of time. (c) The capacitance of the cell loaded with $\mathrm{H}_{2} \mathrm{O} @ \mathrm{C}_{60}$ decreases monotonically due to ortho-para conversion. The capacitances of $(\mathbf{d})$ the empty cell and (e) the cell loaded with $\mathrm{C}_{60}$ change slightly as the temperature is changed, but are constant after the temperature jump. The temperature measurement is shown in $\mathbf{f}$.

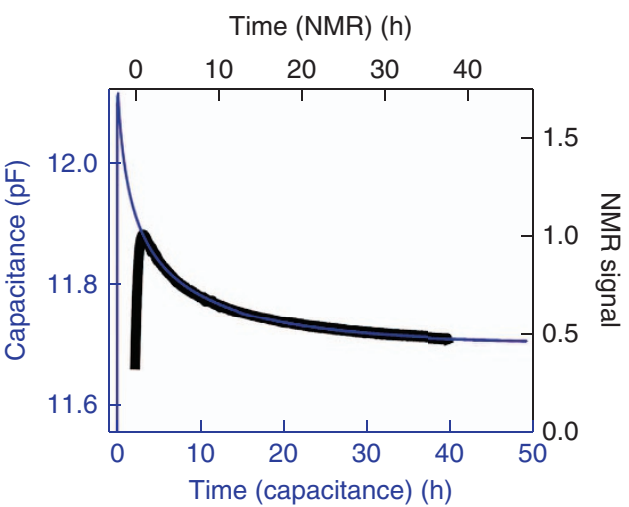

Figure 3 | Comparison with NMR data. Capacitance (blue) and ${ }^{1} \mathrm{H}$ NMR signal (black) as a function of time, measured on the same sample batch. The origins of the capacitance and NMR time axes are defined by the temperature jump.

This difference in initial ortho fraction is due to the slower cooling rate of the NMR probe compared to the capacitance probe, which leads to an initial depletion of the ortho fraction through the enhanced ortho-para conversion rate at high temperature 2,20 . The similar behaviour of the capacitance and NMR data strongly supports the conclusion that the decay in dielectric constant of $\mathrm{H}_{2} \mathrm{O} @ \mathrm{C}_{60}$ is due to nuclear spin isomer conversion (see Supplementary Discussion).

The NMR signal intensity and the dielectric constant both display a rapid initial increase on sample cooling. However these rapid initial changes have a different origin in the two measurements. In NMR, ortho-water gives rise to a signal and para-water does not. The NMR signal of ortho-water depends on the polarization of the Zeeman substates of each ortho level. The Zeeman polarization, and hence the NMR signal, follow the Curie law in this temperature regime and increase on decreasing temperature. As detailed in the Supplementary Discussion and
Supplementary Fig. 4, the dielectric constant also follows a Curie law at higher temperatures, but strong deviations are observed at low temperatures where only a few rotational states are populated.

\section{Discussion}

The dielectric constants of the $\mathrm{H}_{2} \mathrm{O} @ \mathrm{C}_{60} / \mathrm{C}_{60}$ sample and the pure $\mathrm{C}_{60}$ sample may be calculated from the known geometry of the capacitors. From the dielectric constant one can estimate the microscopic molecular polarizability of $\mathrm{H}_{2} \mathrm{O} @ \mathrm{C}_{60}$. We denote the molecular polarizabilities of $\mathrm{C}_{60}$ filled with $\mathrm{H}_{2} \mathrm{O}$ by $\alpha_{\bullet}$ and of empty $\mathrm{C}_{60}$ by $\alpha_{\circ}$, and the corresponding volume densities by $N_{\bullet}$ and $N_{\circ}$, respectively. The molecular polarizabilities are linked to the dielectric constant by the Clausius-Mossotti relation ${ }^{21}$ :

$$
N_{\circ} \alpha_{\circ}+N_{\bullet} \alpha_{\bullet}=3 \epsilon_{0} \frac{(\epsilon-1)}{(\epsilon+2)} .
$$

Knowledge of the empty cage polarizability $\alpha_{\circ}$ and the empty and filled cage volume densities $N_{\circ}$ and $N_{\bullet}$ e enables one to compute $\alpha_{\bullet}$, the molecular polarizability of $\mathrm{H}_{2} \mathrm{O} @ \mathrm{C}_{60}$. To study the effect of the encapsulation of water in $\mathrm{C}_{60}$, we measured the capacitance of the three cells as a function of temperature between 50 and $250 \mathrm{~K}$, and used the Clausius-Mossotti relationship to estimate the molecular polarizabilities for both $\mathrm{C}_{60}$ and $\mathrm{H}_{2} \mathrm{O} @ \mathrm{C}_{60}$. These data are interpreted in terms of a temperature-independent deformation polarizability, plus a temperature-dependent orientational polarizability associated with partial molecular alignment along an applied electric field ${ }^{22}$. In agreement with previous reports ${ }^{23}$ we find a deformation polarizability volume for empty $\mathrm{C}_{60}$ of $\alpha^{\prime}=\alpha /\left(4 \pi \epsilon_{0}\right)$ of $87 \pm 5 \AA^{3}$ and additionally a weak thermally activated polarizability volume of $5 \pm 1 \AA^{3}$ at low temperature $e^{24}$ (see Supplementary Fig. 1 and Supplementary Discussion). For $\mathrm{H}_{2} \mathrm{O} @ \mathrm{C}_{60}$, the molecular polarizability may be written as $\alpha_{\bullet}=\alpha_{\circ}+\alpha_{\bullet}^{\text {orient }}+\Delta \alpha_{\infty}$, where

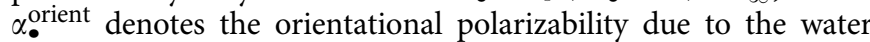
dipole moment and $\Delta \alpha_{\infty}$ corresponds to a change in deformation 
polarizability. The observed change in deformation polarizability is $\Delta \alpha_{\infty}=17 \pm 3 \AA^{3}$; significantly larger than a recent theoretical prediction ${ }^{18}$. The reasons for the discrepancy are currently unknown. The orientational polarizability $\alpha_{\bullet}^{\text {orient }}$, shown in Supplementary Fig. 2, is found to follow a Debye relationship corresponding to a dipole moment of $\mu=0.51 \pm 0.05$ Debye.

The enhanced deformation polarizability of $\mathrm{H}_{2} \mathrm{O} @ \mathrm{C}_{60}$ may be used to calculate its orientational polarizability volume at low temperatures (Supplementary Fig. 3). As detailed in the Supplementary Discussion, the time-dependent capacitance and NMR data may be combined to estimate the individual polarizability volumes of the ortho and para ground states. We obtain $\alpha^{\text {orthol }}=43 \pm 5 \AA^{3}$ and $\alpha^{\text {paral }}=29 \pm 3 \AA^{3}$. These results are in acceptable agreement with the theoretical estimates of $32 \pm 6 \AA^{3}$ and $23 \pm 5 \AA^{3}$ for the ortho and para polarizability volumes of the free rotor states obtained by quantum theory ${ }^{16,25}$, as implemented in the CMIStark software package ${ }^{26}$, taking into account the reduced dipole moment of $0.51 \pm 0.05$ Debye (see Supplementary Fig. 4, Supplementary Table 1 and Supplementary Discussion). The relationship between the orientational polarizability and the free rotor wavefunctions is discussed in ref. 26.

The observed change in the bulk dielectric constant, as encapsulated water converts from the ortho to the para spin isomer, is due to a change in molecular polarizability on spin conversion, with conversion kinetics in agreement with previous NMR studies. Separate molecular polarizabilities for the ortho and para ground states are extracted from the experimental data. The dipole moment of $\mathrm{H}_{2} \mathrm{O} @ \mathrm{C}_{60}$ has been measured experimentally and is found to be in good agreement with recent computational predictions ${ }^{17-19}$. A calculation of the ortho and para ground state polarizabilities based on this dipole moment yields good agreement with the capacitance data. The different response of ortho and para water to electric fields provides a sensitive alternative means to study the spin isomers. The phenomenon opens up the prospect of using Kelvin probe force microscopy $y^{27}$ to study water spin isomers on a single-molecule level.

\section{References}

1. Beduz, C. et al. Quantum rotation of ortho and para-water encapsulated in a fullerene cage. Proc. Natl. Acad. Sci. USA 109, 12894-12898 (2012).

2. Mamone, S. et al. Nuclear spin conversion of water inside fullerene cages detected by low-temperature nuclear magnetic resonance. J. Chem. Phys. 140, 194306 (2014)

3. Goh, K. S. K. et al. Symmetry-breaking in the endofullerene $\mathrm{H}_{2} \mathrm{O} @ \mathrm{C}_{60}$ revealed in the quantum dynamics of ortho and para-water: a neutron scattering investigation. Phys. Chem. Chem. Phys. 16, 21330-21339 (2014).

4. Encrenaz, T. Water in the Solar System. Annu. Rev. Astro. Astrophys. 46, 57-87 (2008).

5. Kravchuk, T. et al. A magnetically focused molecular beam of ortho-water Science 331, 319-321 (2011).

6. Carravetta, M., Johannessen, O. G. \& Levitt, M. H. Beyond the $T_{1}$ limit: singlet nuclear spin states in low magnetic fields. Phys. Rev. Lett. 92, 153003 (2004).

7. Levitt, M. H. Singlet Nuclear Magnetic Resonance. Annu. Rev. Phys. Chem. 63, 89-105 (2012)

8. Farkas, A. Orthohydrogen, Parahydrogen and Heavy Hydrogen. The Cambridge Series of Physical Chemistry (Cambridge University Press, 1935).

9. Andreev, S. N., Makarov, V. P., Tikhonov, V. I. \& Volkov, A. A. Ortho and Para Molecules of Water in Electric Field. Preprint at http://arxiv.org/abs/physics/ 0703038 (2007)

10. Horke, D. A., Chang, Y. P., Długołecki, K. \& Küpper, J. Separating para and ortho water. Angew. Chem. Int. Ed. 53, 11965-11968 (2014).

11. Turgeon, P. A. et al. Preparation, isolation, storage, and spectroscopic characterization of water vapor enriched in the ortho $-\mathrm{H}_{2} \mathrm{O}$ nuclear spin isomer. Phys. Rev. A. 86, 62710 (2012).
12. Redington, R. L. \& Milligan, D. E. Molecular rotation and ortho-para nuclear spin conversion of water suspended in solid Ar, Kr, and Xe. J. Chem. Phys. 39, 1276 (1963).

13. Kurotobi, K. \& Murata, Y. A single molecule of water encapsulated in fullerene C60. Science 333, 613-616 (2011).

14. Krachmalnicoff, A., Levitt, M. H. \& Whitby, R. J. An optimised scalable synthesis of $\mathrm{H}_{2} \mathrm{O} @ \mathrm{C}_{60}$ and a new synthesis of $\mathrm{H}_{2} @ \mathrm{C}_{60}$. Chem. Commun. 50, 13037-13040 (2014).

15. Aoyagi, S. et al. A cubic dipole lattice of water molecules trapped inside carbon cages. Chem. Commun. 50, 524-526 (2014).

16. Bunker, P. R. \& Jensen, P. Molecular Symmetry and Spectroscopy 2nd revised edn (Canadian Science Publishing (NRC Research Press), 2006).

17. Ensing, B., Costanzo, F. \& Silvestrelli, P. L. On the polarity of buckminsterfullerene with a water molecule inside. J. Phys. Chem. A 116 12184-12188 (2012).

18. Galano, A., Pérez-González, A., del Olmo, L., Francisco-Marquez, M. \& León-Carmona, J. R. On the chemical behavior of $\mathrm{C}_{60}$ hosting $\mathrm{H}_{2} \mathrm{O}$ and other isoelectronic neutral molecules. J. Mol. Model 20, 2412-2412 (2014).

19. Sabirov, D. S. Polarizability as a landmark property for fullerene chemistry and materials science. RSC Adv. 4, 44996-45028 (2014).

20. Cacciani, P., Cosléou, J. \& Khelkhal, M. Nuclear spin conversion in $\mathrm{H}_{2} \mathrm{O}$. Phys. Rev. A 85, 012521 (2012).

21. Atkins, P. \& de Paula, J. Atkins' Physical Chemistry (Oxford University Press, 2010).

22. Jackson, J. D. Classical Electrodynamics (Wiley, 1999).

23. Bonin, K. D. \& Kresin, V. V. Electric-dipole Polarizabilities of Atoms, Molecules, and Clusters (World Scientific Publishing Company Incorporated, 1997).

24. Alers, G. B., Golding, B., Kortan, A. R., Haddon, R. C. \& Theil, F. A. Existence of an orientational electric dipolar response in $\mathrm{C}_{60}$ single crystals. Science $\mathbf{2 5 7}$, 511-514 (1992).

25. Zare, R. N. Angular momentum. Understanding Spatial Aspects in Chemistry and Physics (Wiley-Interscience, 1988).

26. Chang, Y. P., Filsinger, F., Sartakov, B. G. \& Küpper, J. CMIstark: Python package for the Stark-effect calculation and symmetry classification of linear, symmetric and asymmetric top wavefunctions in DC electric fields. Comput. Phys. Commun. 185, 339-349 (2014).

27. Nonnenmacher, M., O’Boyle, M. P. \& Wickramasinghe, H. K. Kelvin probe force microscopy. Appl. Phys. Lett. 58, 2921 (1991).

\section{Acknowledgements}

We acknowledge the support of EPSRC-UK (grant number EP/M001962/1), the European Research Council (291044-HYPERSINGLET), and the Wolfson Foundation (WL120007). We thank X. Niu for providing a first version of the PCB spacer. B.M. thanks W. Kossack for useful discussions.

\section{Author contributions}

B.M. constructed the apparatus, performed the experiment, analysed the data and co-wrote the paper. S.M. assisted with the capacitance measurements and data analysis and co-wrote the paper. M.C. and J.A.-V. assisted with capacitance and NMR measurements. A.K. synthesized $\mathrm{H}_{2} \mathrm{O} @ \mathrm{C}_{60}$ guided by R.J.W. and helped with sample preparation. M.H.L. conceived the idea and co-wrote the manuscript.

\section{Additional information}

Supplementary Information accompanies this paper at http://www.nature.com/ naturecommunications

Competing financial interests: The authors declare no competing financial interests.

Reprints and permission information is available online at http://npg.nature.com/ reprintsandpermissions/

How to cite this article: Meier, B. et al. Electrical detection of ortho-para conversion in fullerene-encapsulated water. Nat. Commun. 6:8112 doi: 10.1038/ncomms9112 (2015)

\footnotetext{
This work is licensed under a Creative Commons Attribution 4.0 International License. The images or other third party material in this article are included in the article's Creative Commons license, unless indicated otherwise in the credit line; if the material is not included under the Creative Commons license, users will need to obtain permission from the license holder to reproduce the material. To view a copy of this license, visit http://creativecommons.org/licenses/by/4.0/
} 